\title{
Silicon Cantilever Resonators Integrated with Portable Electrostatic Samplers for Sensing and Characterizing Engineered Nanoparticles in Workplace Air
}

\author{
H. S. Wasisto $^{1^{*}}$, S. Merzsch ${ }^{1}$, A. Waag ${ }^{1}$, I. Kirsch ${ }^{2}$, E. Uhde ${ }^{2}$, T. Salthammer ${ }^{2}$, E. Peiner $^{1}$ \\ ${ }^{1}$ Institute of Semiconductor Technology, TU Braunschweig, Braunschweig, Germany \\ ${ }^{2}$ Material Analysis and Indoor Chemistry Department, Fraunhofer WKI, Braunschweig, Germany \\ ${ }^{*} E$-mail : h.wasisto@tu-bs.de
}

\begin{abstract}
:
A portable cantilever-based nanoparticle detector (CANTOR) is developed and manufactured for sensing engineered nanoparticles (ENPs) in workplace air by monitoring the resonant frequency shift induced by the mass of the particles trapped on the resonator. A piezoresistive Wheatstone bridge and a miniaturized electrostatic sampler are integrated with the cantilever resonator for signal sensing and ENP collection, respectively. By using the first higher harmonic bending frequency, the sensor exhibited a mass sensitivity of $36.51 \mathrm{~Hz} / \mathrm{ng}$, which enables its application to detect airborne carbon ENPs of $\sim 120 \mathrm{~nm}$ in diameter and a concentration of $\sim 6000 \mathrm{NPs} / \mathrm{cm}^{3}$ within a sampling time of $15 \mathrm{~min}$. The electrostatic force generated by reverse-biased $p-n$ junction-patterned substrates is used as a method to position and align ENP delivered from the aerosol phase onto selected regions on the cantilever surface. This developed sensor is targeted for use in handheld airborne ENP-sensing applications.
\end{abstract}

Key words: cantilever resonator, engineered nanoparticle, electrostatic sampler, mass sensitivity.

\section{Introduction}

Nanotechnology has played an important role in the generation of new industrial products and applications through the development of nanoparticles (NPs). However, NPs released in many technological processes can induce occupational hazards to workers. The inhalation of airborne nanoparticles is considered to be the main route of exposure to engineered nanoparticles (ENPs) in the workplace [1]. Therefore, it is essential to monitor personal exposure to ENPs. This intention can be greatly assisted with the use of MEMS based resonators. Such MEMS-based resonant sensors can count the cumulative mass of particles deposited on their surfaces as a shift in their resonant frequencies with high measuring precision. From the previous work, a self-sensing silicon cantilever was investigated in an air pollution sampling of $\sim 20 \mathrm{~nm}$ carbon ENPs which resulted a mass sensitivity of 8.33 $\mathrm{Hz} / \mathrm{ng}$ [2]. Nevertheless, the used commercial nanometer aerosol sampler (TSI 3089) was too bulky and heavy to be implemented in a portable sensing system. Therefore, a lowweight electrostatic sampler was homebuilt to overcome this issue [3]. In this work, a silicon cantilever resonator is integrated with a miniaturized ENP sampler and operated in the first higher harmonic bending to improve the quality factor, mass sensitivity and sampling efficiency in air. Moreover, to select the settlement of ENPs, an electrostatic-directed ENP deposition method is implemented to the novel designed cantilevers which are first tested in an aerosol chamber.

\section{Cantilever Resonator Integrated with Portable Electrostatic Sampler}

A portable cantilever-based nanoparticle detector (CANTOR) is developed to fulfill the demand for personal airborne ENP monitoring. In general, it consists of two main modules, i.e. a cantilever resonator and a miniaturizedelectrostatic sampler (Fig. 1). The first module which is a silicon cantilever resonator (length $I=$ $1.25 \mathrm{~mm}$, width $w=26.5 \mu \mathrm{m}$, height $h=39.3$ $\mu \mathrm{m})$ has a full Wheatstone bridge on its clamped end as a piezoresistive element for reading the signal output of the sensor via a strain-to-resistivity change. The cantilevers are manufactured by utilizing a silicon bulk micromachining process based on photolithography, diffusion, and inductively coupled plasma (ICP) cryogenic deep reactive etching (cryo-DRIE) which offers many benefits including precise control of dimensions, 
integration and miniaturization of devices, and fabrication of an array of devices [2]. An external piezostack adhered to the cantilever supporting frame was used to actuate the cantilever, such that the resonator was able to operate at the first higher harmonic bending frequency $f$ of $221.5 \mathrm{kHz}$ with a quality factor $Q$ of 1950. As it has been known that the quality factor of a resonator increases with the mode number, thus it can help to achieve a higher sensitivity [4].

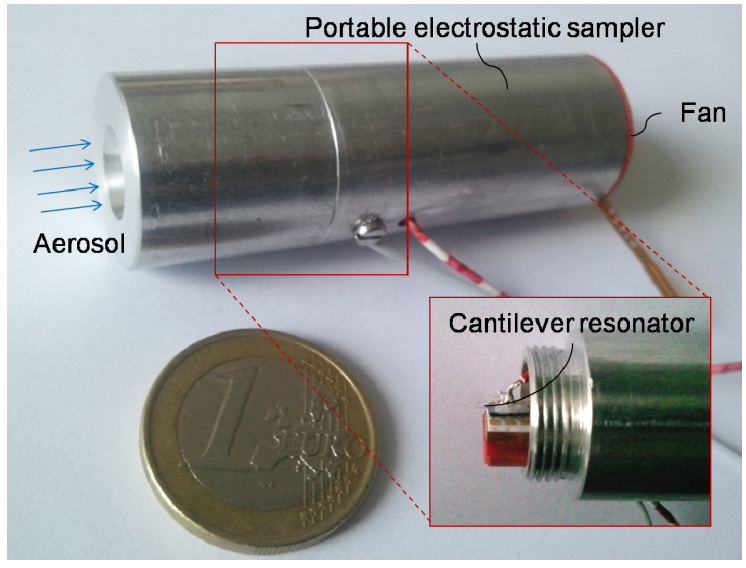

Fig. 1. Portable cantilever-based nanoparticle detector (CANTOR).

The second module which is a miniaturized electrostatic sampler is made of aluminum material. It is designed as a tube with a diameter of $20 \mathrm{~mm}$ and a length of $60 \mathrm{~mm}$ covering the cantilever. The tube consists of three single aluminum parts joined by threads. Those parts are inlet part, middle part, and outlet part. The inlet part is conically shaped with an inclination of $15^{\circ}$ to the tube axis aiming to have a direct aerosol flow against the positioned cantilever. For sucking and generating flow of the aerosol, a fan (MF10A03A, SEPA Europe $\mathrm{GmbH}$ ) is mounted at the outlet part of the tube. Using this fan, a laminar flow $(0.68 \mathrm{l} / \mathrm{min})$ of aerosol is able to pass through the inlet part and directly head to the cantilever. In the middle part of the tube, a silicon cantilever resonator is mounted by double-sided tape on a ceramic printed circuit board (PCB) to integrate it with the electrostatic sampler. Au wire bonding is used for getting electrical contact between cantilever and board. The elastic cable wires soldered to the PCB are then connected to the electronic instruments. After joining inlet and middle parts, the cantilever is located at the center of the conical opening of the inlet. For operating the electrostatic ENP sampler, a portable energy source (i.e. a battery pack with a voltage source of $3.8 \mathrm{~V}$ ) and a DC voltage amplifier converting $3.8 \mathrm{~V}$ into $481 \mathrm{~V}$ are used. High negative voltage is applied to the bulk contact of cantilever. Whereas, the sampler tube wall is grounded to sample the charged or polarized ENPs which are forced in the resulting electrostatic field towards the cantilever. The produced electric fields are dependent on the distance from the center of the circular middle part of tube $(\varnothing=30 \mu \mathrm{m})$ to its wall which has a maximum value of $6500 \mathrm{~V} / \mathrm{mm}$ [3]. ENPs approaching the sensor are encountered by a drastically rising electric field. All components of CANTOR have a total weight of $256 \mathrm{~g}$ and the sampling can be kept stable for 32 hours in case of 2300 mAh battery charge. It offers an advantage compared to TSI 3089 which has a total weight of $3.75 \mathrm{~kg}$. Furthermore, CANTOR does not use any corona dischargers to charge the incoming aerosol as commonly used in many electrostatic precipitators [5]. Instead, it collects the ENPs with their natural charges based on the triboelectric effect.

\section{Carbon-based Engineered Nanoparticle Sampling}

In order to test the functioning of CANTOR, an assessment using carbon-based ENPs was performed in a test chamber under typical workplace condition $\left(V=1 \mathrm{~m}^{3}, T=23^{\circ} \mathrm{C}, \mathrm{rH}=\right.$ $30 \%, p=1 \mathrm{~atm})$. Carbon ENPs were selected as a model system of an engineered aerosol because they are currently used in many industrial applications, e.g. electronic devices, electrochemical devices, filled polymer composites, separation membranes, and drugdelivery systems [6].
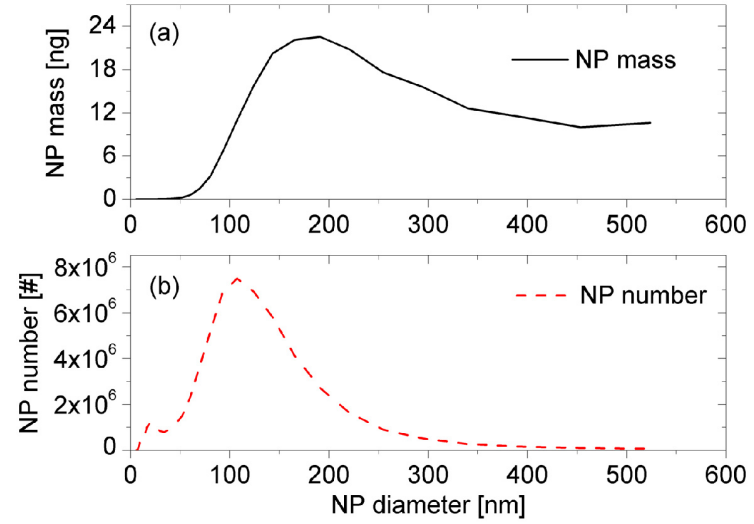

Fig. 2 (a) Mass and (b) number of total carbon ENPs acting upon a silicon cantilever during 15-min sampling as measured by FMPS (TSI 3091). The mass was calculated from the number of carbon ENPs assuming a spherical shape.

A constant output atomizer (TSI 3076) provided an aerosol with defined particle concentration. In situ monitoring was realized by a fast mobility particle sizer (FMPS, TSI 3091). The concentration of total carbon ENPs monitored by FMPS can be further analyzed for determining the particle size distribution during 
sampling. By knowing the flow rate of 680 $\mathrm{cm}^{3} / \mathrm{min}$ and carbon density of $2.267 \mathrm{~g} / \mathrm{cm}^{3}$, the mass and number of the total flowing particles can be determined as $m_{\mathrm{NP}}=203.10 \mathrm{ng}$ and $\mathrm{N}_{\mathrm{NP}}$ $=6.06 \times 10^{7}$ particles, respectively (Fig. 2). During a 15-min carbon ENP sampling at a concentration of $\sim 6000 \mathrm{NPs} / \mathrm{cm}^{3}$, CANTOR is operated to sample ENPs on the whole cantilever surfaces uniformly including cantilever clamped-end and supporting frame as shown in Fig. 3.

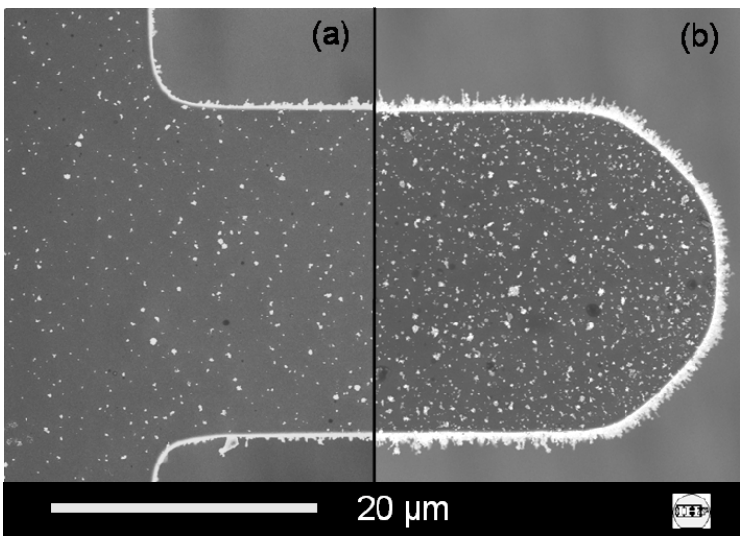

Fig. 3. Loaded cantilever after ENP sampling showing its (a) clamped end and (b) free end.

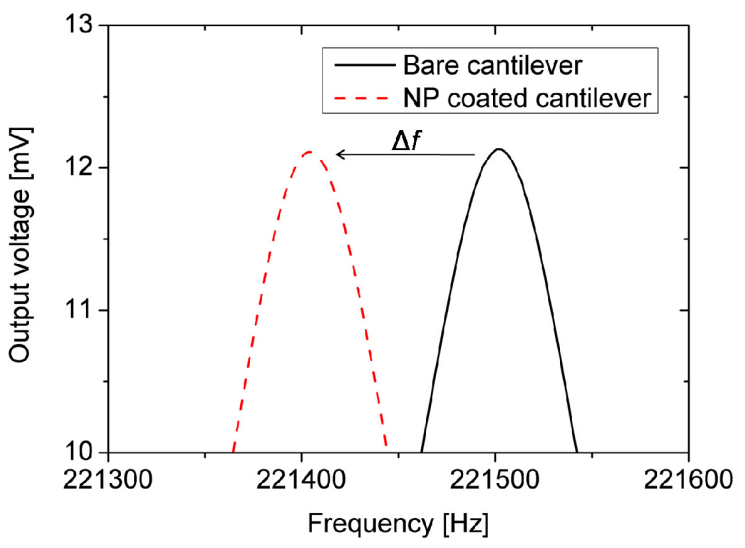

Fig. 4. Measured shift of the first higher harmonic bending frequency induced by the total ENPs' mass adhered to cantilever surface within $15 \mathrm{~min}$.

The resonant frequency shift after airborne ENP sampling is $\Delta f=98.02 \mathrm{~Hz}$ (Fig. 4). With the cantilever mass $m=3.03 \mu \mathrm{g}$, a detected positively charged total ENPs' mass of $\Delta m=2$ $\times m \times \Delta f / f=2.69 \mathrm{ng}$ can be calculated. A mass sensitivity $S=\Delta f / \Delta m=32.75 \mathrm{~Hz} / \mathrm{ng}$ and a sampling efficiency $\eta=\Delta m / m_{\mathrm{NP}} \times 100 \%=$ $1.33 \%$ are therefore yielded from this experiment. This sampling efficiency was improved by a factor of eleven compared to the previous result [2]. Nevertheless, these results are still very low. This considerably high loss of sampling efficiency is because the types of ENPs in generated aerosol are not only positively charged ENPs, but also negatively charged and neutral ENPs. Those negatively charged ENPs were definitely repelled from the cantilever, whereas uncharged and insufficiently polarized ENPs were expected to pass through without being deposited on the cantilever. Moreover, such insufficient electric field intensities might also have contributed to this issue. To compensate for the loss of the sampling efficiencies, flow swirling can be considered in the sampler development, such that airborne ENPs can be exposed to the electric fields as long as possible [7]. A feasibility of collecting both positively and negatively charged ENPs at the same time can also improve the performance of CANTOR.

\section{Selective Nanoparticle Deposition}

Concerning the enhancement of the cantilever resonator module, an electrothermal cantilever is intended to replace the current external piezostack actuator [8]. In this sensor design, ENPs are proposed to be only deposited on a selective area formed by an electrode at the cantilever free end. It is due to the fact that the most sensitive location on the cantilever for detecting micro/nano-sized particles is its freeend [9]. Therefore, motivated by the need of high-sensitive mass detection of ENPs adhered to the microcantilever, a method of electrostatic-directed deposition of ENP on a selective area is investigated using a $p-n$ junction patterned Si substrate [10].

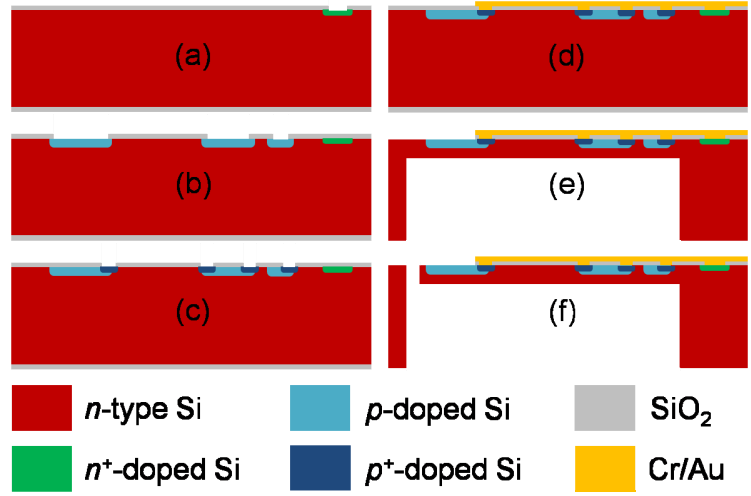

Fig. 5. Fabrication process for electrothermal cantilever resonators.

The fabrication processes of the resonators (Fig. 5) were begun with phosphorus diffusion in $n$-type bulk silicon wafers $(1-10 \Omega \mathrm{cm})$ of 300 $\mu \mathrm{m}$ in thickness. $\mathrm{A} \mathrm{SiO}_{2}$ layer of $300 \mathrm{~nm}$ served as a mask (a). Subsequently, boron diffusion was used to form a p-type ENP-collection electrode, heaters and a Wheatstone bridge, respectively (b). Additional boron diffusion was performed to create $p^{+}$-regions and improve contact formation (c). Metallization was carried out by evaporation of $\mathrm{Cr} / \mathrm{Au}(30 / 300 \mathrm{~nm})$ which was patterned in a lift-off process (d). Backside 
etching of membrane was done by ICP cryo DRIE with photoresist of $1.7 \mu \mathrm{m}$ serving as the etching mask (e). Finally, the cantilevers were lithographically patterned and released by frontside ICP cryo-DRIE (f).

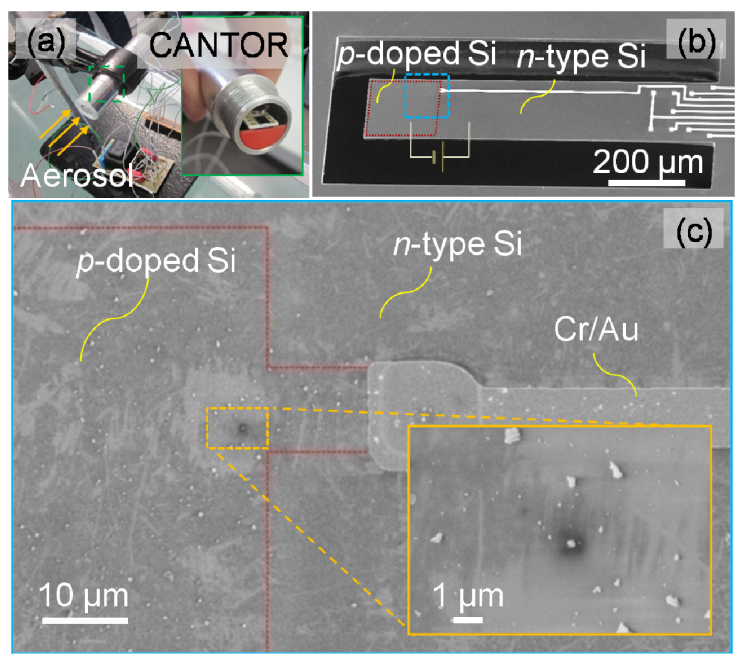

Fig. 6. (a) Carbon aerosol sampling setup, (b) resulting selective ENP deposition, and (c) a magnified view on the $p$-doped Si area.

The first test of this electrostatic-directed ENP deposition method was performed utilizing a carbon aerosol (concentration $=\sim 4500$ $\mathrm{NP} / \mathrm{cm}^{3}$, sampling time $=2 \mathrm{hrs}$ ) which was not precharged, i.e. consisted of positively and negatively charged and neutral ENPs (Fig. 6(a)). Using CANTOR, upon reverse bias an additional electric field is established in the charge depletion zone. From Fig. 6 (b and c), a number of positively charged carbon ENPs is clearly deposited uniformly on the p-type Si region, indicating that positively charged particles were being repelled by the $n$-type $\mathrm{Si}$ region and attracted to the $p$-type $S i$ region. When the positively charged particles get closer to the substrate they are expected to experience stronger attracting or repelling forces. A positive voltage of the ENP sampler tube has also created additional electrostatic forces which push the positively charged ENPs down to the substrate and keep the negatively charged ENPs away from the substrate. From FMPS monitoring, the total number and mass of the ENPs forced to flow through CANTOR are found to be $5.4 \times 10^{8}$ particles and $2.15 \mu \mathrm{g}$, respectively. In regard to the collected ENPs of $7.8 \times 10^{4}$ counted by observing SEM images with per viewing area $(10 \mu \mathrm{m} \times 7.5 \mu \mathrm{m})$ at a magnification of $10 \mathrm{kX}$, a NP collection efficiency of $0.014 \%$ is obtained. This relatively low collection efficiency can be attributed to the excessive distance between the incoming particle and the substrate. Thus, most particle motions were governed by Brownian motion instead of electrostatic forces as a result of a weak interaction between the incoming particles and the substrate. The laminar flow of aerosol which was parallel to the substrate may also have influenced the effectiveness of this method. Therefore, the trajectory, collection area, and electric fields have to be further optimized to improve the collection efficiency.

\section{Conclusion}

The performance of a portable cantilever-based nanoparticle detector (CANTOR) for the detection of airborne ENPs has been shown in this work. Sampling efficiency improvement was obtained comparing to our previous results with a commercial bulky sampler. For real-time ENP monitoring, work is in progress to integrate a resonant frequency tracking circuit (e.g. phaselocked loop and microcontroller). Electrostaticdirected deposition was demonstrated using $p-n$ junction patterned Si substrate of cantilever.

\section{Acknowledgements}

The authors thank J. Arens, D. Rümmler, K. H. Lachmund, and S. Wientzek for their technical assistances. This work is performed in the collaborative project "NanoExpo" funded by the German Federal Ministry of Education and Research (BMBF) within the cluster "NanoCare" under no. 03X0098A.

\section{References}

[1] J. W. Card, D. C. Zeldin, J. C. Bonner, and E. R. Nestmann, Am J Physiol Lung Cell Mol Physiol 295: L400-L411 (2008)

[2] H. S. Wasisto, S. Merzsch, A. Waag, I. Kirsch, E. Uhde, T. Salthammer, E. Peiner, Microsyst. Technol. (2012); doi:10.1007/s00542-011-1405-9

[3] S. Merzsch, H. S. Wasisto, A. Waag, I. Kirsch, E. Uhde, T. Salthammer, E. Peiner, Proc. IEEE Sensors 2011 Conf., pp. 1177-1180 (2011); doi:10.1109/ICSENS.2011.6127335

[4] M. K. Ghatkesar, V. Barwich, T. Braun, J-P. Ramseyer, C. Gerber, M. Hegner, H. P. Lang, U. Drechsler and M. Despont, Nanotechnology 18, 445502 (8pp) (2007); doi:10.1088/09574484/18/44/445502

[5] E.M.Kettleson, B.Ramaswami, C.J.Hogan Jr., M.H.Lee, G.A.Statyukha, P.Biswas, L.T.Angenent, Environ. Sci. Technol. 43 5940-5946 (2009)

[6] E. G. Rakov, Russian Chemical Reviews 70 (10) 827-863 (2001)

[7] J. W. Lee, S. H. Lee, J. Jang, Journal of Electrostatics 1-9 (2012); doi:10.1016/j.elstat.2011.12.005

[8] H. S. Wasisto, S. Merzsch, A. Waag, I. Kirsch, E. Uhde, T. Salthammer, E. Peiner, Proc. IEEE Sensors 2011 Conf., pp. 736-739 (2011); doi:10.1109/ICSENS.2011.6127061

[9] S. Dohn, R. Sandberg, W. Svendsen, A. Boisen, Applied Physics Letters 86, 233501 (2005)

[10] D-H. Tsai, T. Hawa, H-C. Kan, R. J. Phaneuf and M. R. Zachariah, Nanotechnology 18, 365201 (10pp) (2007) 